\title{
TITLE:
}

\section{Up-stream dispatching of power by density of power packet}

$\operatorname{AUTHOR}(S)$ :

Nawata, Shinya; Takahashi, Ryo; Hikihara, Takashi

\section{CITATION:}

Nawata, Shinya ... [et al]. Up-stream dispatching of power by density of power packet. IEICE Transactions on Fundamentals of Electronics, Communications and Computer Sciences 2016, E99A(12): 2581-2584

ISSUE DATE:

2016-12-01

URL:

http://hdl.handle.net/2433/217760

\section{RIGHT:}

Copyright (c) 2016 The Institute of Electronics, Information and Communication Engineers. 


\title{
LETTER \\ Up-Stream Dispatching of Power by Density of Power Packet
}

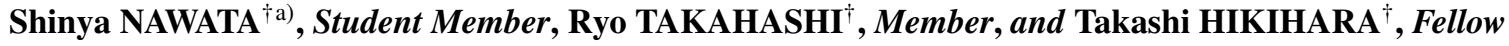

SUMMARY Power packet is a unit of electric power transferred by a pulse with an information tag. This letter discusses up-stream dispatching of required power at loads to sources through density modulation of power packet. Here, power is adjusted at a proposed router which dispatches power packets according to the tags. It is analyzed by averaging method and numerically verified.

key words: power packet, up-stream dispatching, router, power density

\section{Introduction}

In sharing energy between sources and loads in an electrical energy network, there exist up-stream dispatching of required power to sources and down-stream dispatching of supplied power to loads [1]. With information and communication technologies, the network can possibly adjust the best matching of supplies and demands in on-demand manners [2], [3]. However, with physically coupled sources and loads, it is apparently difficult to realize the desired supplies and demands simultaneously in the network. Thus, the key is to directly and physically process the power in the network. To this end, we introduce power packetization.

Power packet is defined as a unit of electric power transferred by a pulse with an information tag. The concept of power packet was proposed from the viewpoint of suppliers by Toyoda and Saitoh in 1990s [4]. "Energy packet networks" were also proposed to provide energy on demand to Cloud Computing servers [5]. Recently, widebandgap power semiconductor devices, which have the potential to realize higher frequency switching and higher power capacity than Si power devices, have been developed [6], [7]. The authors' group has developed a power packet dispatching system in physical layer applying these highperformance power semiconductor devices [8]-[11]. In the system, power packets are directly and physically tagged as their voltage waveforms. The system consists of network lines and routers which forward power packets according to the tags. Energy storage is integrated into the router to adjust the forwarding of power packets. It is also confirmed that the router realizes power transfer using timedivision multiplexing (TDM) without keeping synchronization of clocks for generating packets between routers*. Considering the pair of information tags and power pulses as a symbol, power packet transmission is found as a problem

\footnotetext{
Manuscript received June 1, 2016.

The authors are with the Department of Electrical Engineering, Kyoto University, Kyoto-shi, 615-8510 Japan.

a)E-mail: nawata@ dove.kuee.kyoto-u.ac.jp

DOI: 10.1587/transfun.E99.A.2581
}

of simultaneous representation of message and energy using symbol sequences in a similar way to the Shannon's information theory [12].

The up-stream and down-stream dispatching of power can be dynamically realized by routing power based on the tags of packets. Here is handled the power as the density of power packet [13]. Up to now, the method for generating power packets is proposed to realize desired dynamical behavior of the objective loads with dynamic quantizer for discrete-valued signal [14], [15]. The aim of downstream dispatching is to transfer limited amount of supplied power to multiple loads. On the other hand, the aim of upstream dispatching is to transfer required power from multiple sources in the desired amount of power.

In this letter, we focus on the up-stream dispatching of required power at loads to sources through density modulation of power packet in transmission. It is discussed at a router, i.e. a unit element of the network which dispatches power packets spatially and temporally based on information tags. Here, we show the relationship between transferred power into the router and density of packets in transmission. The relationship is analyzed by averaging method [16] and numerically simulated taking dynamics of the storage integrated into the router into account.

\section{Up-stream Dispatching of Power at Router}

\subsection{Power Packetization and Function of Router}

Here, we explain the configuration of power packet and the function of router based on our developed system [9]. A power packet consists of header, payload, and footer. The payload brings power, while the header and the footer carry the information of the packet's tag. The information tag identifies varieties of power due to different sources, loads, voltages, and so on.

A router is designed for multiple inputs, outputs, and storages implemented with capacitors. The schematic configuration of the router is shown in Fig. 1. Varieties of power, which are identified at lines by tags' information, are identified in the router by multiple storages. Thus, varieties of power can be separately and individually managed in a single network.

*The packet itself contains the preamble for recognizing the sent information and fixing the clock of receiving router [10], [11]. 


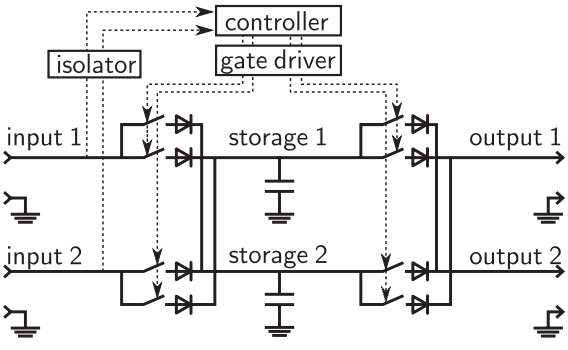

Fig. 1 Schematic configuration of the router with two inputs, two outputs, and two storages [9].

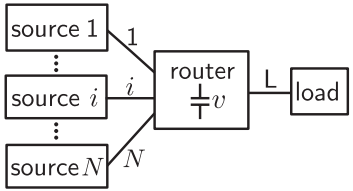

Fig. 2 Schematic diagram of system to investigate up-stream dispatching of power at router.

\subsection{Problem Setting}

With handling power as continuous flow by averaging [13], this letter focuses on up-stream dispatching of power packets stored at a same storage in each router. Power packets are physically coupled if they are stored at a same storage. Except it, there is no coupling. In the up-stream dispatching, a router is supplied with required power of loads from multiple sources and through multiple passes.

The up-stream dispatching is investigated at the setting in Fig. 2. In the system, power packets are asynchronously transferred to the same storage through multiple inputs. The input ports are indexed by a finite set $I=\{1,2, \cdots, N\}$ and the output port is indexed by "L". The power packets transferred at the port $i \in I$ to the storage is called packet $i$ and power packets transferred at the port $\mathrm{L}$ from the storage is called packet L.

Considering a finite duration, $u_{i}$ denotes the average power transferred by packet $i$ for $i \in I \cup\{\mathrm{L}\} . \sigma_{i}$ denotes density of packet $i$ in transmission, i.e. the proportion of the payload of the packet $i$. For a time series $x,\langle x\rangle$ denotes the average of $x$. For packet $i$ with non-empty payload, $\langle x\rangle_{i}$ denotes the average of $x$ over the payload of the packet $i$. Considering a stationary state, the problem of up-stream dispatching is treated by the proportion of each transferred power $\left\{u_{i}\right\}_{i \in I}$ in the total supplied power $\sum_{i \in I} u_{i}$.

Finally, the operation of the system is explained as follows. For $i \in I$, the source of the packet $i$ is treated as a Thévenin equivalent circuit with open-circuit voltage $e_{i} \geq 0$ and resistance $r_{i}$. The support of $e_{i}$ corresponds to the payload of packet $i$. By definition, $\left\langle e_{i}\right\rangle=\sigma_{i}\left\langle e_{i}\right\rangle_{i}$. As for the router, the storage capacitance is set as $C$ and the voltage across the storage is denoted by $v$. A diode is placed at each switch to protect the reverse current as in Fig. 1, so that the reverse current is prevented at the time of $e_{i}<v$ for each $i \in I$. The router regenerates the packet $\mathrm{L}$ with the voltage equal to $v$ during the payload. The load is treated as resistance $R$ with PFC circuit, which requires energy $u_{\mathrm{R}}=\sigma_{\mathrm{L}} E_{0}^{2} / R$, where $E_{0}$ is a base value of voltage ${ }^{\dagger}$.

\section{Analysis by Averaging Method}

Here, we analyze the relationship between transferred power into the router and the density of packets in transmission. In the analysis, we assume small ripple fluctuation of the voltage $v$. According to the averaging method [16], we approximate $v \approx\langle v\rangle$.

At first, we consider a small ripple fluctuation of $e_{i}$ for $i \in I$ and a small difference between $\left\{e_{i}\right\}_{i \in I}$ enough to have $e_{i}>v$ for all $i \in I$. The packet $i$ transfers power approximated by

$$
u_{i} \approx \sigma_{i}\langle v\rangle \frac{\left\langle e_{i}\right\rangle_{i}-\langle v\rangle}{r_{i}}
$$

In addition, the following equation is led by averaging the constraint equation of the Kirchhoff's current law at the router's storage in a stationary state.

$$
\sum_{i \in I} \sigma_{i} \frac{\left\langle e_{i}\right\rangle_{i}-\langle v\rangle}{r_{i}} \approx \sigma_{\mathrm{L}} \frac{\langle v\rangle}{R}
$$

or

$$
\langle v\rangle \approx \frac{\sum_{i \in I} \frac{\sigma_{i}}{r_{i}}\left\langle e_{i}\right\rangle_{i}}{\sum_{i \in I} \frac{\sigma_{i}}{r_{i}}+\frac{\sigma_{L}}{R}}
$$

Using Eqs. (1) and (3), we can derive the proportion of the transferred power by packet $j$ for each $j \in I$ :

$$
\frac{u_{j}}{\sum_{i \in I} u_{i}} \approx \frac{\frac{\sigma_{j}}{r_{j}}\left\langle e_{j}\right\rangle_{j}+\sum_{i \in I} \frac{R}{\sigma_{\mathrm{L}}} \frac{\sigma_{j}}{r_{j}} \frac{\sigma_{i}}{r_{i}}\left(\left\langle e_{j}\right\rangle_{j}-\left\langle e_{i}\right\rangle_{i}\right)}{\sum_{i \in I} \frac{\sigma_{i}}{r_{i}}\left\langle e_{i}\right\rangle_{i}}
$$

If differences at source sides are negligible, the densities of power packets give the proportion of each transferred power. In other words, if $\left\langle e_{i}\right\rangle_{i}=\left\langle e_{j}\right\rangle_{j}$ and $r_{i}=r_{j}$ for all $i, j \in I$, the proportion can be written as

$$
\frac{u_{j}}{\sum_{i \in I} u_{i}} \approx \frac{\sigma_{j}}{\sum_{i \in I} \sigma_{i}}
$$

This relationship shows the possibility of dynamical adjustment of the up-stream dispatching of power at routers in a network through density modulation of power packet.

Finally, we briefly discuss the case $e_{i}<v$ for some $i \in I$. During $e_{i}<v$, no power is transferred by packet $i$, because the diode at link $i$ prevents reverse instantaneous power [11]. Therefore, the effect of the diodes should be taken into account by using power density at port $i^{\dagger \dagger}$ instead

${ }^{\dagger}$ In the whole network, the base value of voltage may be different depending on the varieties of power identified by tags and storages [9].

${ }^{\dagger}$ Power density at port $i$ is the proportion of support of instantaneous power at port $i$, which is included in the payload of packet $i$. The power density at link $i$ takes a value between 0 to $\sigma_{i}$. 
of $\sigma_{i}$ which is the density of packet $i$ in transmission.

\section{Numerical Verification}

The analytical results in Sect. 3 are obtained with the small ripple fluctuation of the voltage $v$ across the capacitor. In this section, the relationship between transferred powers and packet densities is numerically verified by simulating the dynamics of the voltage $v$. For simplicity, we consider the case in which the number of inputs are two, i.e. $I=\{1,2\}$. As an example, the basis of the system is set by $E_{0}=12 \mathrm{~V}$ and $u_{\mathrm{R}}=1 \mathrm{~W}$. Each packet is periodically transmitted with the period from $0.5 \mathrm{~ms}$ to $5 \mathrm{~ms}{ }^{\dagger}$. The payload of packet 1 and packet 2 has rectangular waveform of voltage, i.e. $e_{i}=\left\langle e_{i}\right\rangle_{i}$ on the payload for $i \in\{1,2\}$. We set $C=1000 \mu \mathrm{F}$ and $r_{1}=r_{2}=1 \Omega$. The transferred powers $u_{1}$ and $u_{2}$ are defined in a duration of $100 \mathrm{~ms}$.

In the following, the transferred powers $\left\{u_{i}\right\}_{i \in I}$ are calculated in various settings of densities $\left\{\sigma_{i}\right\}_{i \in I}$ in the both cases of $\left\langle e_{1}\right\rangle_{1}=\left\langle e_{2}\right\rangle_{2}$ and $\left\langle e_{1}\right\rangle_{1} \neq\left\langle e_{2}\right\rangle_{2}$. The period and the phase of each packet are uniformly distributed. The density $\sigma_{\mathrm{L}}$ is uniformly distributed from 0 to 1 , and then the resistance of the load is decided as $R=\sigma_{\mathrm{L}} \cdot E_{0}^{2} / u_{\mathrm{R}}=\sigma_{\mathrm{L}} \cdot 144 \Omega$. The switches and diodes in the router are ideal in the simulation.

\subsection{In Case of $\left\langle e_{1}\right\rangle_{1}=\left\langle e_{2}\right\rangle_{2}$}

At first, we discuss the case of $\left\langle e_{1}\right\rangle_{1}=\left\langle e_{2}\right\rangle_{2}=E_{0}$. The relationship between transferred powers and packet densities is examined in the simulation by uniformly distributing $\sigma_{1}$ and $\sigma_{2}$ from 0 to 1 . The results of 10,000 trials are plotted in Figs. 3 and 4. Figure 3 shows that, almost regardless of the dynamics, the densities of power packets give the proportion of each transferred power as in Eq. (5).

Figure 4 shows that the total required power is satisfied with the sufficiently large sum of densities, but is not satisfied with the small sum of densities. Here, using Eq. (3), the total transferred power can be approximated by averaging method as

$$
u_{1}+u_{2} \approx \frac{\langle v\rangle^{2}}{R}=\left(\frac{\sigma_{1}+\sigma_{2}}{\sigma_{1}+\sigma_{2}+0.0069}\right)^{2} \mathrm{~W}
$$

This relationship, shown in Fig. 4 as a solid line, approximates the simulated results well. Thus, the sum of densities should be set sufficiently large in order to keep the voltage across the capacitor close to $E_{0}$ and supply sufficient power.

\subsection{In Case of $\left\langle e_{1}\right\rangle_{1} \neq\left\langle e_{2}\right\rangle_{2}$}

Next, we discuss the case of $\left\langle e_{1}\right\rangle_{1} \neq\left\langle e_{2}\right\rangle_{2}$. As an example, we set $\left\langle e_{1}\right\rangle_{1}=12.5 \mathrm{~V}$ and $\left\langle e_{2}\right\rangle_{2}=12 \mathrm{~V}$. The relationship

${ }^{\dagger}$ In this simulation, the time series of packet $\mathrm{L}$ is assumed to be independent from the time series of inputted packets. In our developed router, the packet $\mathrm{L}$ can be regenerated based on the time series of inputted packets by router's algorithms [9].

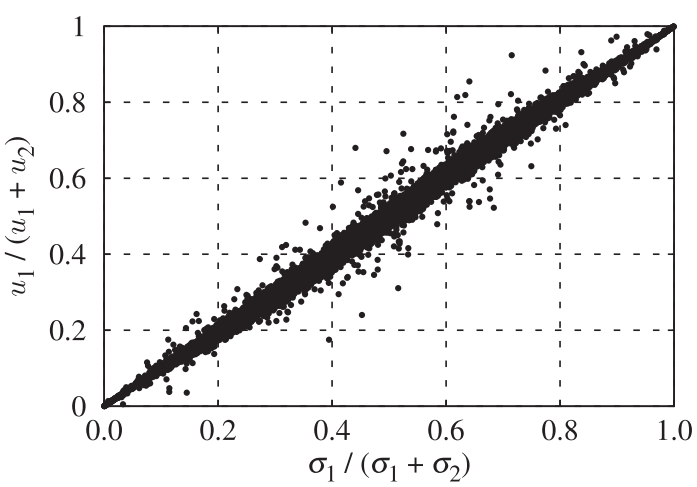

Fig. 3 Relationship between proportion of transferred power by packet 1 and packet densities at $\left\langle e_{1}\right\rangle_{1}=\left\langle e_{2}\right\rangle_{2}=E_{0}$.

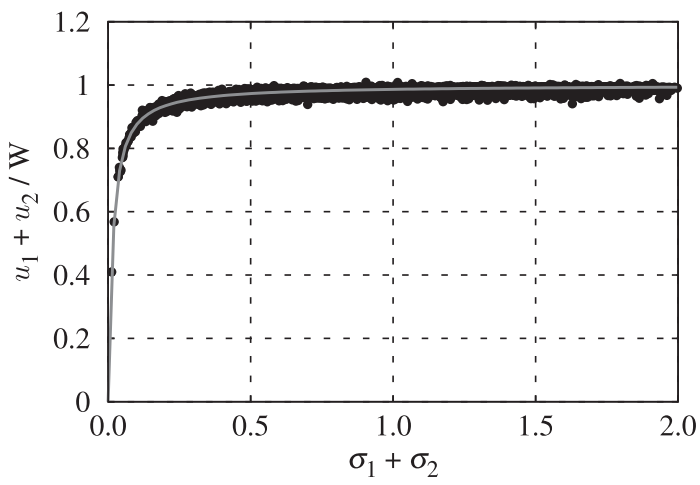

Fig. 4 Relationship between total amount of transferred power and packet densities at $\left\langle e_{1}\right\rangle_{1}=\left\langle e_{2}\right\rangle_{2}=E_{0}$. The solid line shows the relationship of Eq. (6).

between transferred powers and packet densities is numerically examined again by uniformly distributing $\sigma_{1}$ and $\sigma_{2}$ from 0 to 1 . The simulation was repeated 10,000 times and the results were drawn in Figs. 5 and 6. In Fig. 6, to ignore the effect of the voltage drop, only the results with the sufficiently large sum of densities are plotted.

Figure 5 shows no power is transferred by packet 2 if $\sigma_{1}$ is not small. This means the power density at link 2 becomes 0 for all possible values of $\sigma_{2}$ if packet 1 is frequently transmitted. Fig. 5, however, also shows the proportion of transferred power by packet 2 ranges from 0 to 1 if $\sigma_{1}$ is sufficiently small. In addition, Fig. 6 shows, if the sum of densities is sufficiently large, i.e. $\sigma_{1}+\sigma_{2}>0.3$ in this case, the required power is satisfied ${ }^{\dagger \dagger}$. These results imply, even if the difference between source voltages is not small, we can adjust the proportion of input power by setting small the densities of packets with high voltage in transmission.

Using 10 different seeds for the random number generator, similar simulation results are obtained with Figs. 3, 4, 5 , and 6 , which ensure the consistency of the results.

${ }^{\dagger}$ The transferred power slightly decreases when $\sigma_{1}$ is small. The result implies that the supplied voltage varies from $\left\langle e_{1}\right\rangle_{1}$ to $\left\langle e_{2}\right\rangle_{2}$. 


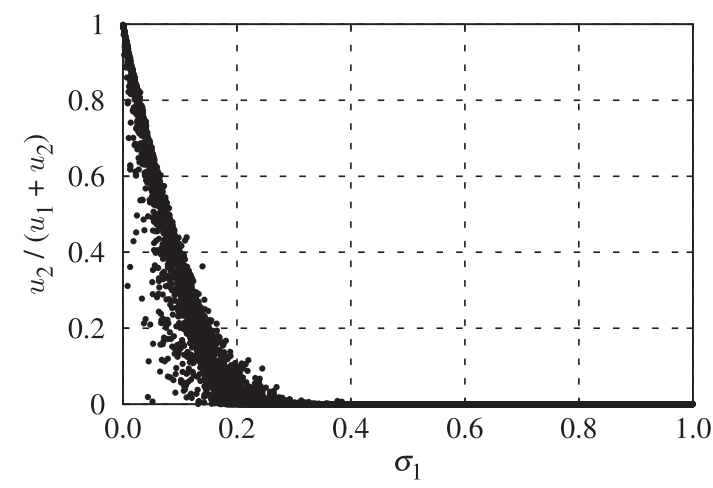

Fig. 5 Relationship between proportion of transferred power by packet 2 and density of packet 1 at $\left.\left\langle e_{1}\right\rangle_{1}\right\rangle\left\langle e_{2}\right\rangle_{2}$.

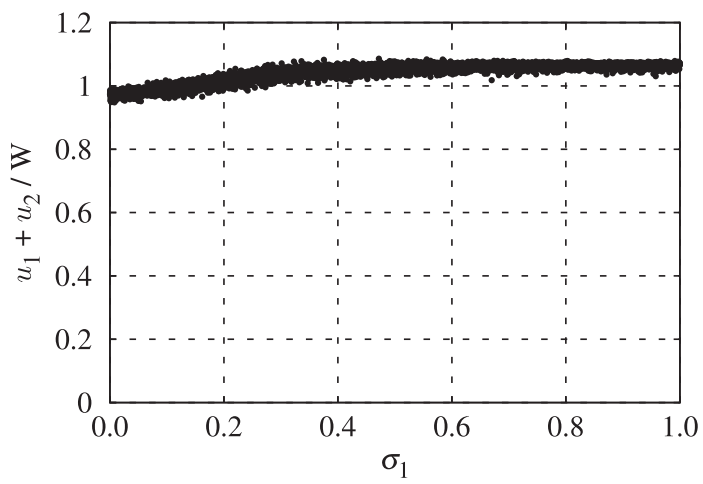

Fig. 6 Relationship between total amount of transferred power and density of packet 1 at $\left.\left\langle e_{1}\right\rangle_{1}\right\rangle\left\langle e_{2}\right\rangle_{2}$. Only the results which satisfy $\left.\sigma_{1}+\sigma_{2}\right\rangle$ 0.3 are plotted to ignore the voltage drop across the capacitor.

\section{Concluding Remarks}

This letter demonstrated the possibility of realizing the upstream dispatching of required power to the sources by density modulation of power packet. The up-stream dispatching is adjustable at the router with power packets transferred through multiple inputs to the storage. The averaging method reveals the relationship between density of power packets and transferred power by the packets. By numerically simulating the dynamics of the router's storage, it is verified that the up-stream dispatching can be realized if the total density of inputted packets is set sufficiently large. Simulated results clearly show that even if the difference between voltages of packets is not small, the dispatching is realized by reducing the densities of packets with high voltage in transmission. These discussions provide a method for realizing desired supplies and demands by processing power by power packetization through electrical energy networks.

\section{Acknowledgment}

This work was partially supported by the Cross-ministerial
Strategic Innovation Promotion Program from New Energy and Industrial Technology Development Organization (NEDO) and by the Super Cluster Program (Kyoto) from Japan Science and Technology Agency. The author (R.T.) was partially supported by the JSPS, Grant-in-Aid for Young Scientist (B), 26820144.

\section{References}

[1] J. Bialek, "Tracing the flow of electricity," IEE Proc., Gener. Transm. Distrib., vol.143, no.4, pp.313-320, July 1996.

[2] K. Sakai and Y. Okabe, "Quality-aware energy routing toward ondemand home energy networking: (Position paper)," 2011 IEEE Consumer Communications and Networking Conference (CCNC), pp.1041-1044, Jan. 2011.

[3] T. Yoshihisa, N. Fujita, and M. Tsukamoto, "A rule generation method for electrical appliances management systems with home EoD," Proc. 1st IEEE Global Conference on Consumer Electronics 2012, pp.248-250, 2012.

[4] J. Toyoda and H. Saitoh, "Proposal of an open-electric-energynetwork (OEEN) to realize cooperative operations of IOU and IPP," Proc. 1998 International Conference on Energy Management and Power Delivery, EMPD'98, pp.218-222, 1998.

[5] E. Gelenbe, "Energy packet networks: Adaptive energy management for the cloud," Proc. 2nd International Workshop on Cloud Computing Platforms, CloudCP'12, pp.1-5, 2012.

[6] T. Funaki, J.C. Balda, J. Junghans, A.S. Kashyap, H.A. Mantooth, F Barlow, T. Kimoto, and T. Hikihara, "Power conversion with SiC devices at extremely high ambient temperatures," IEEE Trans. Power Electron., vol.22, no.4, pp.1321-1329, July 2007.

[7] T. Takuno, T. Hikihara, T. Tsuno, and S. Hatsukawa, "HF gate drive circuit for a normally-on SiC JFET with inherent safety," Proc. 13th European Conference on Power Electronics and Applications, pp.14, Barcelona, Spain, Sept. 2009.

[8] T. Takuno, M. Koyama, and T. Hikihara, "In-home power distribution systems by circuit switching and power packet dispatching," Proc. 2010 First IEEE International Conference on Smart Grid Communications, pp.427-430, 2010.

[9] R. Takahashi, K. Tashiro, and T. Hikihara, "Router for power packet distribution network: Design and experimental verification," IEEE Trans. Smart Grid, vol.6, no.2, pp.618-626, March 2015.

[10] Y. Zhou, R. Takahashi, N. Fujii, and T. Hikihara, "Power packet dispatching with second-order clock synchronization," Int. J. Circ. Theor. Appl., vol.44, no.3, pp.729-743, March 2016.

[11] Y. Zhou, Power packet dispatching based on synchronization with features on safety, Ph.D. dissertation, Department of Electrical Engineering, Kyoto University, 2015.

[12] S. Nawata, R. Takahashi, and T. Hikihara, "An asymptotic property of energy representation with power packet," IEICE Trans. Fundamentals (Japanese Edition), vol.J97-A, no.9, pp.584-592, Sept. 2014.

[13] T. Hikihara, "Power processing by packetization and routing," IEICE Technical Report, CCS2015-57, Nov. 2015.

[14] R. Takahashi, S. Azuma, K. Tashiro, and T. Hikihara, "Design and experimental verification of power packet generation system for power packet dispatching system," Proc. 2013 American Control Conference, pp.4368-4373, 2013.

[15] S. Mochiyama, N. Fujii, R. Takahashi, and T. Hikihara, "A study on trajectory control of manipulator using power packet dispatching," Proc. 2015 IEEE 37th International Telecommunications Energy Conference, pp.555-559, Osaka, Japan, Oct. 2015.

[16] J.G. Kassakian, M.F. Schlecht, and G.C. Verghese, Principles of Power Electronics, pp.261-274, Addison-Wesley, USA, 1991. 